\title{
Angioedema due to acquired C1-inhibitor deficiency: spectrum and treatment with C1-inhibitor concentrate
}

\author{
Konrad Bork ${ }^{1 *}$ (D) Petra Staubach-Renz ${ }^{1}$ and Jochen Hardt ${ }^{2}$
}

\begin{abstract}
Background: Acquired angioedema due to $\mathrm{C} 1$-inhibitor (C1-INH) deficiency (AAE-C1-INH) is a serious condition that may result in life-threatening asphyxiation due to laryngeal edema. It is associated with malignant B-cell lymphoma and other disorders. The purpose of this study was to describe the characteristics and associated disorders of patients with AAE-C1-INH and assess the efficacy of plasma-derived C1-INH concentrate (pdC1-INH) in the treatment of AAE-C1INH. Forty-four patients with AAE-C1-INH from the Angioedema Outpatient Service of Mainz were assessed for associated disorders. In 32 of these patients, the duration of swelling attacks was measured before and after treatment with pdC1INH (Berinert ${ }^{\oplus}$ (CSL Behring, Marburg, Germany)). The time between injection and complete resolution of symptoms and treatment effectiveness was provided by the patients.
\end{abstract}

Results: The following underlying disorders were present: monoclonal gammopathy of undetermined significance (47.7\%), non-Hodgkin lymphoma (27.3\%), anti-C1-INH autoantibodies alone (11.4\%), and other conditions (4.5\%). In $9.1 \%$ patients, no associated disorder could be found. AAE-C1-INH led to the detection of lymphoma in $75 \%$ of patients with the malignancy. Treatment with pdC1-INH shortened attacks by an average (SD) $54.4( \pm 32.8)$ hours $(P<0.0001)$. The earlier the attack was treated, the shorter the time between injection and resolution of symptoms $(P=0.0149)$. A total of $3553(97.7 \%)$ of the 3636 attacks were effectively treated with pdC1-INH as assessed by the patient. The mean (SD) dose per-attack was 787 ( \pm 442) U. pdC1-INH was effective in 1246 (93.8\%) of 1329 attacks in 8 patients with anti-C1-INH autoantibodies and in 344 (99.4\%) of 346 attacks in 6 patients without autoantibodies. The average (SD) dose per effectively treated attack was 1238.4 ( \pm 578.2) $\mathrm{U}$ in patients with anti-C1-INH autoantibodies and $510.2( \pm 69.1) \cup$ in patients without autoantibodies.

Conclusions: pdC1-INH is highly effective in treating AAE-C1-INH patients and is also effective in the vast majority of attacks in patients with anti-C1-INH autoantibodies. It is fast-acting and reduces attack duration.

Keywords: Acquired angioedema, C1-inhibitor deficiency, Non-Hodgkin lymphoma, C1-inhibitor concentrate, C1-inhibitor antibodies

\section{Background}

Acquired angioedema due to C1-inhibitor (C1-INH) deficiency (AAE-C1-INH) or angioedema due to acquired C1-INH deficiency is characterized by acquired deficiency of C1-INH, recurrent angioedema, and hyperactivation of the complement pathway. Patients have recurrent swellings or attacks of the skin (face, extremities, and genitals) and severe abdominal attacks, sometimes with diarrhea and

\footnotetext{
*Correspondence: konrad.bork@unimedizin-mainz.de

1 Department of Dermatology, Johannes Gutenberg University,

Langenbeckstr. 1, 55131 Mainz, Germany

Full list of author information is available at the end of the article
}

vomiting, due to edema of the gastrointestinal mucosa. They may also present with potentially life-threatening edema of the upper respiratory tract, oral mucosa and tongue. Death by asphyxiation has been reported [1-3]. Attacks usually last from 2 to 5 days without any apparent trigger [4]. There is no epidemiological data for AAE-C1-INH available but prevalence has been estimated to range between 1:100,000 and 1:500,000 [4].

AAE-C1-INH may be associated with B-cell abnormalities such as malignant lymphoma, monoclonal gammopathy of undetermined significance (MGUS), and anti-C1-INH autoantibodies. Although functional $\mathrm{C} 1-\mathrm{INH}$ is produced in

(c) The Author(s). 2019 Open Access This article is distributed under the terms of the Creative Commons Attribution 4.0 International License (http://creativecommons.org/licenses/by/4.0/), which permits unrestricted use, distribution, and 
AAE-C1-INH, it is thought that these associated disorders can lead to a deficiency of the C1-INH protein [5-8]. The deficiency of C1-INH leads to inappropriate activation of the contact-kinin system, release of bradykinin, increased vascular permeability and angioedema $[9,10]$.

Diagnosis of AAE-C1-INH generally occurs after 40 years of age [11]. There is no genetic association or family history of angioedema in AAE-C1-INH; this is in contrast to the types of hereditary angioedema (HAE) which are caused by or associated with specific mutations in the genes coding for $\mathrm{C} 1-\mathrm{INH}$, factor XII, plasminogen or angiopoietin [12-15]. Patients with AAE-C1-INH have low plasma levels of C1-INH (functional and antigenic) and $\mathrm{C} 4$ which are usually below $50 \%$ of normal. Unlike HAE-C1-INH patients, the majority of AAE-C1-INH patients also have reduced $\mathrm{C} 1 \mathrm{q}$ levels. The presence of anti-C1-INH autoantibodies and the absence of C1-INH genetic mutations can aid in the diagnosis of AAE-C1-INH [4].

Currently, there is no approved treatment for AAE-C1-INH. HAE-C1-INH therapies such as plasma-derived C1-INH concentrate (pdC1-INH) and the bradykinin B2 receptor antagonist, icatibant, are used to resolve AAE-C1-INH attacks [16]. It is generally supposed that these on-demand treatments are most effective when administered early in the attack [17]. However, published information on the efficacy and safety of AAE-C1-INH treatments is limited and has not been studied systematically.

Therefore, the aim of this observational, retrospective study was to: (1) describe the characteristics and associated disorders of patients with AAE-C1-INH, (2) assess the efficacy of pdC1-INH in the treatment of AAE-C1-INH patients (3) assess the effect of time to injection, dose and anti-C1-INH autoantibodies on pdC1-INH efficacy.

\section{Results}

\section{Patient characteristics}

Patients with a confirmed diagnosis of AAE-C1-INH attending our outpatient clinic, Department of Dermatology, University of Mainz, Germany (AOSM), were eligible for this observational study. In total, 44 patients with AAE-C1-INH were identified and their health records were reviewed and analyzed. The prevalence of AAE-C1-INH compared with that of HAE-C1-INH in the AOSM was 1:9.3. The majority (61\%) of patients were female and mean (SD) age at onset of recurrent angioedema of AAE-C1-INH was $56.2( \pm 14.8)$ years (Table 1). Five $(11.4 \%)$ of the 44 patients were aged under 40 years when the recurrent angioedema started. Two female patients had onset at 21 and 30 years and had anti-C1-INH autoantibodies; another male and female patient had onset at 37 and 39 years, respectively and had MGUS. The fifth patient had no associated disorders. In these 5 patients, molecular genetic testing for a mutation in SERPING1 was negative.

Clinical symptoms were abdominal attacks, skin swellings of the face, extremities and genitals as well as isolated tongue swellings and laryngeal attacks. An erythema marginatum preceded the symptoms in 2 patients. Seven patients died during the course of the study; 2 deaths were from underlying diseases. There were no angioedema-related deaths in this group.

\section{Associated disorders}

The search for associated disorders in all 44 AAE-C1-INH patients revealed the following (Table 1):

(1) MGUS was associated with 21 (47.7\%) patients: $\operatorname{IgG}(n=12), \operatorname{IgM}(n=6)$, and $\operatorname{IgA}(n=3)$. The mean (SD) age at onset of angioedema in patients with MGUS was $58.5( \pm 13.5)$ years.

(2) Non-Hodgkin lymphoma was present in $12(27.3 \%)$ patients. Of these, $11(25.0 \%)$ patients had low-grade malignancy lymphoma: splenic marginal cell lymphoma $(n=6)$, plasmocytoma $(n=2)$, B-cell lymphoma $(n=1)$, Waldenström's macroglobulinemia $(n=1)$, and centroblastic-centrocytic follicular lymphoma $(n=1)$. One (2.3\%) patient had high-grade malignancy lymphoma: diffuse anaplastic large cell B-cell lymphoma. Six of the 12 patients with non-Hodgkin lymphoma presented with monoclonal gammopathies: $\operatorname{IgG}(n=3)$ and $\operatorname{IgM}(n=3)$. The mean (SD) age at onset of angioedema in patients with non-Hodgkin lymphoma was $62.4( \pm 12.3)$ years.

(3) Five $(11.4 \%)$ patients had anti-C1-INH autoantibodies and, besides recurrent angioedema, they had no other underlying associated disorder. In these patients, recurrent angioedema started at ages $21,30,40,50$, and 54 years giving a mean (SD) age at onset of $39( \pm 13.7)$ years. Interestingly, patients with anti-C1-INH autoantibodies and no other disorder were notably younger at the onset of angioedema than patients with MGUS (58.5 ( \pm 13.5$)$ years) or malignant lymphoma (62.4 ( \pm 12.3) years). During an observation period of mean (SD) 15.8 $( \pm 9.4)$ years following anti-C1-INH autoantibody detection, no MGUS, lymphoma or other underlying disorders besides autoantibodies were found. An additional 3 patients had anti-C1-INH autoantibodies and an underlying associated disorder of MGUS $(n=2)$ and diffuse anaplastic large cell B-cell lymphoma $(n=1)$.

(4) Two (4.5\%) patients had other associated disorders: breast cancer $(n=1)$ and liver failure $(n=1)$.

(5) The $4(9.1 \%)$ remaining patients had no associated disorders such as MGUS, non-Hodgkin lymphoma, anti-C1-INH autoantibodies, cancer or liver failure. Three of the 4 patients had low C1-INH activity and protein, low $\mathrm{C} 4$ and low $\mathrm{C} 1 \mathrm{q}$ in plasma. One of the 4 patients, a female, had recurrent angioedema of the 
Table 1 Patient characteristics

\begin{tabular}{|c|c|c|c|c|}
\hline \multirow[b]{2}{*}{ Age at first angioedema (years), mean (SD) } & \multicolumn{2}{|c|}{$\begin{array}{l}\text { All patients } \\
(N=44)\end{array}$} & \multicolumn{2}{|c|}{$\begin{array}{l}\text { pdC1-INH treated patients } \\
(N=32)\end{array}$} \\
\hline & 56.2 & $(14.8)$ & 56.0 & $(14.7)$ \\
\hline < 40 (years), n (\%) & 5 & $(11.4)$ & 4 & $(12.5)$ \\
\hline $40-<50$ (years), $n(\%)$ & 9 & $(20.4)$ & 6 & (13.6) \\
\hline $50-<60$ (years), n (\%) & 11 & $(11.0)$ & 7 & (21.9) \\
\hline $60-<70$ (years), n (\%) & 10 & $(22.7)$ & 9 & $(28.1)$ \\
\hline $70-<80$ (years), n (\%) & 7 & $(15.9)$ & 4 & $(12.5)$ \\
\hline$\geq 80$ (years), n (\%) & 2 & $(4.5)$ & 2 & (6.3) \\
\hline \multicolumn{5}{|l|}{ Gender, n (\%) } \\
\hline Male & 17 & (38.6) & 14 & $(43.8)$ \\
\hline Female & 27 & $(61.4)$ & 18 & $(56.3)$ \\
\hline \multicolumn{5}{|l|}{ Associated disorders, n (\%) } \\
\hline MGUS & 21 & $(47.7)$ & 15 & $(46.9)$ \\
\hline Non-Hodgkin lymphoma & 12 & $(27.3)$ & 10 & $(31.3)$ \\
\hline Splenic marginal cell lymphoma & 6 & $(13.6)$ & 4 & $(12.5)$ \\
\hline Plasmocytoma & 2 & $(4.5)$ & 2 & (6.3) \\
\hline B-cell lymphoma & 1 & $(2.3)$ & 1 & (3.1) \\
\hline Waldenström's macroglobulinemia & 1 & $(2.3)$ & 1 & (3.1) \\
\hline Centroblastic-centrocytic follicular lymphoma & 1 & $(2.3)$ & 1 & $(3.1)$ \\
\hline Diffuse anaplastic large cell B-cell lymphoma & 1 & $(2.3)$ & 1 & (3.1) \\
\hline Anti-C1-INH autoantibodies with no other associated disorders ${ }^{a}$ & 5 & $(11.4)$ & 5 & (15.6) \\
\hline Other associated disorders & 2 & $(4.5)$ & 0 & \\
\hline None & 4 & (9.1) & 2 & (6.3) \\
\hline \multicolumn{5}{|l|}{ Plasma complement, n (\%) } \\
\hline \multicolumn{5}{|l|}{ C1-INH function } \\
\hline$<$ normal range of $70-130 \%$ & 44 & $(100)$ & 32 & $(100)$ \\
\hline$<50 \%$ & 44 & $(100)$ & 32 & $(100)$ \\
\hline$<5 \%$ & 28 & $(63.6)$ & 21 & (65.6) \\
\hline \multicolumn{5}{|l|}{ C1-INH protein } \\
\hline$<$ normal range of $15.4-33.8 \mathrm{mg} / \mathrm{dL}$ & 44 & $(100)$ & 32 & $(100)$ \\
\hline$<12 \mathrm{mg} / \mathrm{dL}$ & 41 & $(93.2)$ & 32 & $(100)$ \\
\hline$<4.8 \mathrm{mg} / \mathrm{dL}$ & 27 & 61.4 & 20 & (62.5) \\
\hline \multicolumn{5}{|l|}{ C4 } \\
\hline$<$ normal range of $16.4-31.3 \mathrm{mg} / \mathrm{dL}$ & 44 & $(100)$ & 32 & $(100)$ \\
\hline$<12 \mathrm{mg} / \mathrm{dL}$ & 43 & $(97.7)$ & 32 & (100) \\
\hline$<4.8 \mathrm{mg} / \mathrm{dL}$ & 39 & $(88.6)$ & 30 & (93.8) \\
\hline \multicolumn{5}{|l|}{$\mathrm{Clq}$} \\
\hline$<$ normal range of $0.1-0.25 \mathrm{~g} / \mathrm{L}$ & 39 & $(88.6)$ & 29 & $(90.6)$ \\
\hline$<0.05 \mathrm{~g} / \mathrm{L}$ & 26 & $(59.1)$ & 21 & (65.6) \\
\hline
\end{tabular}

${ }^{\mathrm{a}}$. an additional 3 patients had autoantibodies to $\mathrm{C} 1-\mathrm{INH}$ and an associated disorder $\mathrm{C} 1-\mathrm{INH}=\mathrm{C} 1$ inhibitor; pdC1-INH = plasma-derived C1-inhibitor concentrate; MGUS = monoclonal gammopathy of undetermined significance; $\mathrm{SD}=$ standard deviation

extremities and lips and abdominal attacks for 17 years (from age 25 to 42 years). During this time, the patient had low C1-INH function and protein and low $\mathrm{C} 4$ but normal C1q. For the last 4 years, the patient has had no clinical symptoms and all 6 tests for C1-INH function and protein and $\mathrm{C} 4$ revealed normal results. Mutations 
in the SERPING1 gene could not be identified and there was no family history of angioedema. The patient's parents had normal C1-INH function and protein.

In 18 patients, recurrent angioedema was present more than 1 year before an associated disorder was diagnosed, in 23 patients both were simultaneously diagnosed and in 3 patients, recurrent angioedema developed later than the associated disorder. In 9 (75.0\%) of 12 patients, the associated malignant lymphoma was detected following diagnosis of AAE-C1-INH. In the other 3 patients, the underlying lymphoma was already known before the first visit at the AOSM.

\section{Plasma complement}

All 44 patients had functional and antigenic C1-INH plasma levels which were below the normal range (Table 1). C4 levels were also below the normal range in all patients; C1q levels were below normal levels in 39 (88.6\%) patients.

\section{Prior and concomitant treatment}

Prior to initiation of treatment with pdC1-INH (Berinert ${ }^{\circ}$ (CSL Behring, Marburg, Germany)), patients received prophylactic therapy with oral prophylaxis: danazol (10 (22.7\%) patients), tranexamic acid (10 (22.7\%) patients) and antihistamines (1 (2.3\%) patients). Acute attacks were treated with icatibant (11 (25.0\%) patients), cortisone (10 $(22.7 \%)$ patients) and antihistamines (2 (4.5\%) patients). Rituximab (5 (11.4\%) patients) and clexanes (2 (4.5\%) patients), were administered for the long-term treatment of swellings. Patients spent on average $2.8( \pm 4.5)$ stays in hospital and $0.82( \pm 2.7)$ days in intensive care for the treatment of acute attacks before pdC1-INH treatment. Three patients had one intubation each and 2 patients had 2 intubations each. Three patients underwent a cricothyrotomy procedure.

During the pdC1-INH treatment period, patients continued to occasionally use icatibant to treat acute attacks (11 (25.0\%) patients). Rituximab (5 (11.4\%) patients) and clexanes (2 (4.5\%) patients) were also administered during the pdC1-INH treatment phase.

\section{Plasma-derived C1-inhibitor concentrate treatment Attacks, treatment duration and dose}

The pdC1-INH treatment group comprised 32 patients, the characteristics of which are listed in Table 1. A total of 1962 attacks were experienced in this group before diagnosis in the abdomen $(785(40 \%))$, face $(580(29.6 \%))$, extremities $(504(25.7 \%))$, genitals $(36(1.8 \%))$, tongue $(35(1.8 \%))$, and larynx $(22(1.1 \%))$. These patients received pdC1-INH for a mean $(\mathrm{SD}) 82.6( \pm 80.1)$ months. They were treated for 3636 attacks mainly occurring in the abdomen (2522 (56.1\%)), face (954 (21.2\%)), and extremities (650 (14.5\%)) (Table 2 and Fig. 1). A smaller number $(367$ (8.2\%)) of attacks occurring in the genitals, tongue and larynx were also treated. pdC1-INH was administered at the $500 \mathrm{U}$ dose for 2203 attacks and at the $1000 \mathrm{U}$ dose for 1095 attacks (Table 2). A total of 338 attacks were treated with higher doses of $1500 \mathrm{U}, 2000 \mathrm{U}$ or $3000 \mathrm{U}$.

\section{Efficacy of pdC1-INH concentrate treatment}

The mean (SD) duration of untreated attacks was 89.9 $( \pm 14.8)$ hours over all attacks in the 32 patients and 84 $( \pm 31.8)$ hours on a per-patient basis (mean of 32 averages). The mean (SD) duration of the treated attacks was $27.9( \pm 12.9)$ hours over all attacks in the 32 patients and 29.6 ( \pm 16.4) hours on a per-patient basis. (Fig. 2). Treatment with pdC1-INH concentrate shortened attacks by an average of $54.4( \pm 32.8)$ hours (confidence interval $(\mathrm{CI})$ : $42.5,66.2)(P<0.0001)$ on a per-patient basis (i.e. by $64.8 \%$ ).

All 32 patients responded to treatment, with 12 (37.5\%) patients experiencing relief in $30 \mathrm{~min}$ or less. The average time to first relief after injection was $1.0( \pm 3.3)$ hour in all 32 patients and $3.6 \mathrm{~h}( \pm 8.2)$ on a per-patient basis. Resolution of symptoms occurred on average 26.4 ( \pm 12.6) hours after injection in all 32 patients and $26.7( \pm 15.0)$ hours on a per-patient basis.

A total of 3553 (97.7\%) of the 3636 attacks were effectively treated as assessed by the patient. Eighty-eight (2.3\%) attacks in 8 patients did not or did not sufficiently respond to pdC1-INH treatment. Among the 8 non-responders were 4 patients with anti-C1-INH autoantibodies (described further below).

\section{Effect of time to injection on efficacy}

The mean (SD) time between attack onset and injection was $1.5( \pm 0.9)$ hours for the 32 patients and $3.0( \pm 2.7)$ hours on a per-patient basis. Regression analysis showed that there was a linear association between the average time to complete resolution of symptoms and the average time to injection i.e. the earlier the attack was treated, the shorter the time between injection and resolution of symptoms $(P=0.0149)$. Sensitivity analysis, omitting 2 patients with extreme times to injection, confirmed the association $(P=0.0036)$. Similar regression analysis showed no clear association between the average shortening of attack duration and the time to injection $(P=0.0745)$.

\section{Effect of dose on efficacy}

In order to analyze the effect of dose, a subgroup analysis of patients using either $500 \mathrm{U}$ or $\geq 1000 \mathrm{U}$ in at least $90 \%$ of their acute injections was performed (Table 3). Overall, the mean $(\mathrm{SD})$ dose per-attack was $787( \pm 442) \mathrm{U}$. The majority of patients received either $500 \mathrm{U}(n=13)$ or 1000 $\mathrm{U}(n=9)$ in more than $90 \%$ of attacks; 1 patient received $1500 \mathrm{U}$ and 1 patient received $2000 \mathrm{U}$. Patients in both 
Table 2 Attacks treated with plasma-derived C1-inhibitor concentrate

\begin{tabular}{|c|c|c|c|}
\hline & \multicolumn{3}{|c|}{$\begin{array}{l}\text { pdC1-INH concentrate treated attacks } \\
(N=32)\end{array}$} \\
\hline & Total & Mean & (SD) \\
\hline Attacks treated with pdC1-INH concentrate, $n$ & 3636 & 113.6 & $(336.2)$ \\
\hline \multicolumn{4}{|l|}{ Attacks treated by location, $\mathrm{n}$} \\
\hline Abdominal attacks & 2522 & 78.8 & $(275.9)$ \\
\hline Facial attacks & 954 & 29.8 & $(115.5)$ \\
\hline Extremity attacks & 650 & 20.3 & $(65.8)$ \\
\hline Genital attacks & 255 & 8.0 & $(43.5)$ \\
\hline Tongue attacks & 71 & 2.2 & $(9.5)$ \\
\hline Laryngeal attacks & 41 & 1.3 & (3.4) \\
\hline \multicolumn{4}{|l|}{ Attacks treated by dose, $\mathrm{n}$} \\
\hline $500 \mathrm{U}$ & 2203 & 68.8 & $(300.7)$ \\
\hline $1000 U$ & 1095 & 34.2 & $(144.9)$ \\
\hline $1500 U$ & 22 & 0.7 & $(2.2)$ \\
\hline $2000 U$ & 315 & 9.8 & $(53.5)$ \\
\hline $3000 \mathrm{U}$ & 1 & 0 & $(0.2)$ \\
\hline
\end{tabular}

pdC1-INH = Plasma-derived C1-inhibitor; $S D=$ Standard deviation

dose subgroups had similar body weights and mean times between attack onset and injection (Table 3).

In the $500 \mathrm{U}$ dose group, attack duration was shortened by a mean (SD) 53.1 ( \pm 25.9) hours and in the $\geq 1000$ U dose group by a mean (SD) 58.8 ( \pm 40.1 ) hours. In the $500 \mathrm{U}$ dose group, the time between injection and resolution of symptoms was $27.6( \pm 16.3)$ hours and in the $\geq 1000 \mathrm{U}$ dose group it was 21.9 ( \pm 11.3 ) hours. However, no association between dose and shortening of attack duration $(P>0.1)$ or dose and time between injection and resolution of symptoms $(\mathrm{P}>0.1)$ could be shown by linear regression.

\section{Effect of anti-C1-INH autoantibodies on efficacy}

Eight AAE-C1-INH patients were positive for anti-C1-INH autoantibodies and 6 patients were negative. The remainder of patients were not tested for the presence of anti-C1-INH autoantibodies.

Attack duration was shortened by a mean (SD) 50.4 ( \pm 38.2) hours in the 8 patients who tested positive for

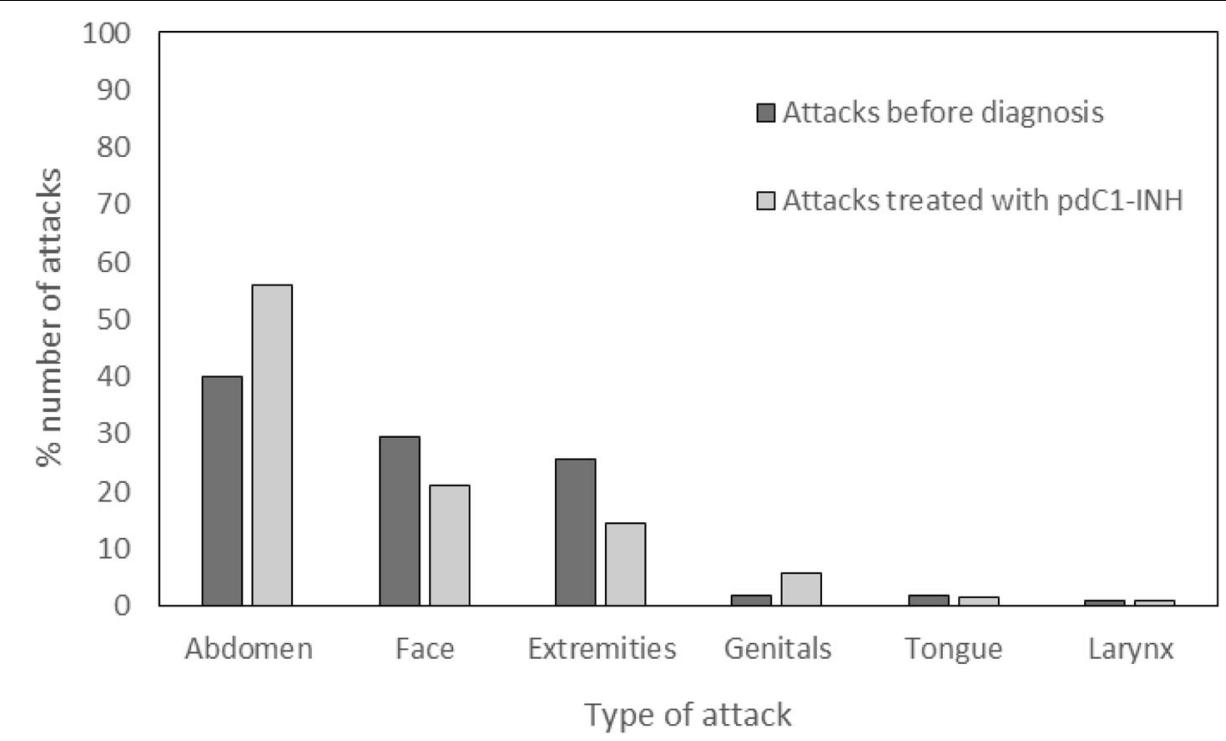

Fig. 1 Location of attacks in acquired angioedema C1-inhibitor patients before diagnosis and after treatment with plasma-derived C1-inhibitor concentrate pdC1-INH = plasma-derived C1-inhibitor. 


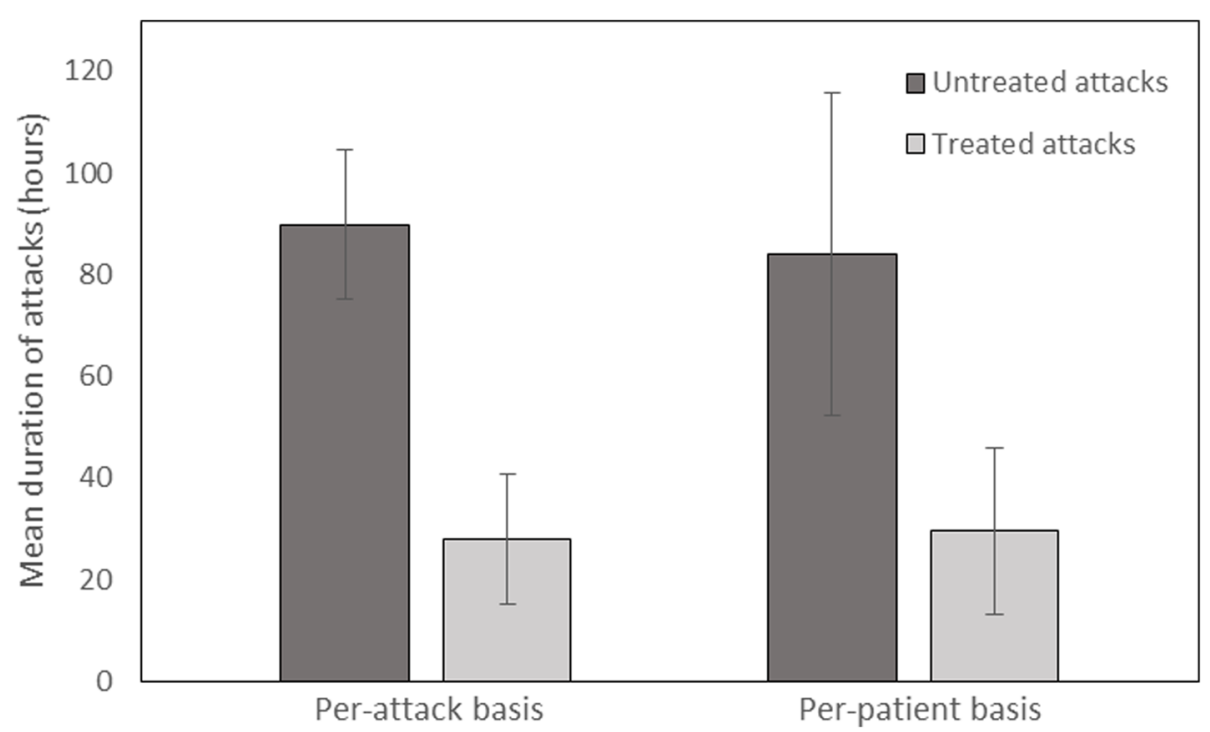

Fig. 2 Efficacy of plasma-derived C1-inhibitor concentrate in acquired angioedema due to C1-inhibitor deficiency. Mean (SD) duration of untreated versus treated attacks is shownSD $=$ standard deviation.

antibodies to C1-INH and by $58.9 \mathrm{~h}( \pm 36.0)$ in the $6 \mathrm{pa}-$ tients who tested negative. The mean shortening of attack duration for each individual patient with anti-C1-INH autoantibodies is shown in Table 4. The mean (SD) time between injection and resolution of symptoms was $31.5( \pm 21.1)$ hours in the antibody positive patients and $29.3( \pm 17.5)$ hours in the antibody negative patients.

Table 4 shows the efficacy in the subgroup of patients with anti-C1-INH autoantibodies. The 8 antibody-positive patients had a total of 2963 attacks: 1634 were untreated and 1329 were treated with pdC1-INH. In all 8 patients, pdC1-INH was effective in 1246 attacks (93.8\%). In 4 of the 8 patients, pdC1-INH was not effective in 83 attacks. These 4 patients had 95 further attacks which were effectively treated with pdC1-INH.

Five of the 8 anti-C1-INH autoantibody positive patients (patients 1, 2, 3, 7, 8) had no associated disorders such as MGUS or malignant lymphoma. These patients experienced 986 attacks, 971 (98.5\%) of which were effectively treated, as assessed by the patient.
Table 5 shows the treatment effect by dose in the subgroups of patients with and without anti-C1-INH autoantibodies. In 6 patients without anti-C1-INH autoantibodies, 344 (99.4\%) of 346 attacks were treated effectively, with an average (SD) dose of $510.2( \pm 69.1) \mathrm{U}$ per effectively treated attack. In 8 patients with anti-C1-INH autoantibodies, 1246 (93.8\%) of the 1329 attacks were treated effectively, with an average (SD) dose of 1238.4 ( \pm 578.2) U per effectively treated attack. In 4 of the 8 anti-C1-INH autoantibody positive patients, 83 attacks failed to respond or did not sufficiently respond to pdC1-INH. Sixty-nine $(83.1 \%)$ of those 83 attacks were treated with $500 \mathrm{U}$. A dose of $500 \mathrm{U}$ did, however, effectively treat $33(32.4 \%)$ of the total 102 attacks.

\section{Discussion}

The clinical features of our patients diagnosed with AAE-C1-INH were similar to those described in previous AAE-C1-INH studies [1, 4, 18]. In our institution, we found that the incidence of the condition was $1 \mathrm{pa}-$ tient with AAE-C1-INH for every 9.3 patients with

Table 3 Effect of dose on plasma-derived C1-inhibitor concentrate efficacy

\begin{tabular}{lll} 
& $\begin{array}{l}\geq 1000 \cup^{a} \\
(N=11)\end{array}$ \\
\hline Body weight, $\mathrm{kg}$ & $(\mathrm{N}=13)$ & Mean (SD) \\
Time between attack onset and injection, hours & $68.8(24.0)$ & $70.8(12.6)$ \\
Shortening of attack with treatment, hours & $2.3(1.1)$ & $3.3(4.2)$ \\
Time between injection and resolution of symptoms, hours & $53.1(25.9)$ & $58.8(40.1)$ \\
\hline
\end{tabular}

adose received in at least $90 \%$ of administrations

$\mathrm{SD}=$ standard deviation 
Table 4 Treatment efficacy in acquired angioedema C1-inhibitor patients with anti-C1-INH autoantibodies $(N=8)$

\begin{tabular}{|c|c|c|c|c|c|c|c|c|}
\hline Patient & $\begin{array}{l}\text { Gender } \\
(\mathrm{M} / \mathrm{F})\end{array}$ & $\begin{array}{l}\text { Type of anti-C1-INH } \\
\text { autoantibodies }\end{array}$ & $\begin{array}{l}\text { Untreated } \\
\text { attacks } \\
\text { (n) }\end{array}$ & $\begin{array}{l}\text { Mean duration of } \\
\text { untreated attacks } \\
\text { (h) }\end{array}$ & $\begin{array}{l}\text { Treated } \\
\text { attacks } \\
\text { (n) }\end{array}$ & $\begin{array}{l}\text { Mean duration of } \\
\text { treated attacks a } \\
\text { (h) }\end{array}$ & $\begin{array}{l}\text { Mean shortening of attacks } \\
\text { with treatment }(h,(\%))\end{array}$ & $\begin{array}{l}\text { Effectively } \\
\text { treated attacks } \\
\text { b } \\
(\mathrm{n}(\%))\end{array}$ \\
\hline 1 & $F$ & $\lg G$ & 124 & 72 & 820 & 9.5 & $62.5(86.8)$ & $820(100)$ \\
\hline 2 & $M$ & $\lg G, \lg M$ & 208 & 60 & 13 & 79 & $-19(-31.7)$ & $1(8.3)$ \\
\hline 3 & $M$ & $\lg G$ & 648 & 84 & 12 & 41.5 & $42.5(50.6)$ & $12(100)$ \\
\hline 4 & M & $\lg G, \lg M$ & 79 & 96 & 16 & 26 & 70 (72.9) & $16(100)$ \\
\hline 5 & F & $\lg A$ & 89 & 84 & 24 & 38 & $46(54.8)$ & $22(91.7)$ \\
\hline 6 & M & $\lg G$ & 59 & 96 & 303 & 24.25 & 71.75 (74.7) & $303(100)$ \\
\hline 7 & $M$ & $\lg G, \lg M$ & 144 & 120 & 71 & 11 & 109 (90.8) & 70 (98.6) \\
\hline 8 & F & $\lg G, \lg M$ & 283 & 72 & 70 & 51.5 & $20.5(28.5)$ & 68 (97.1) \\
\hline Total & - & - & 1634 & - & 1329 & - & - & 1246 (93.8) \\
\hline
\end{tabular}

atime between onset of attack and injection + time between injection and resolution of symptoms

bas assessed by the patient

C1-INH = C1-inhibitor; $F=$ Female; $I g=$ Immunoglobulin; $M=$ Male

HAE-C1-INH. In other studies, an incidence of 1:8.8 [1], $6 \%$ [19] and 10\% [18] was reported. The clinical picture of our AAE-C1-INH patients differs from a large series of HAE-C1-INH patients previously described by us [20]. AAE-C1-INH patients have (1) a higher number of facial swellings (29.6\% versus $1.6 \%)$, (2) a lower number of extremity swellings ( $25.7 \%$ versus $45.1 \%$ ), and (3) a higher number of tongue swellings ( $1.8 \%$ versus $0.3 \%)$ than the HAE-C1-INH patients. The difference in the swelling patterns between AAE-C1-INH and HAE-C1-INH shows that a low level of functional C1-INH activity alone does not determine the swelling pattern. Furthermore, the number of patients with preceding erythema marginatum is lower in AAE-C1-INH patients $(4.5 \%)$ than in HAE-C1-INH patients (30 to 60\%) $[21,22]$.

In most patients in our series, onset of AAE-C1-INH occurred at age 40 years or older. However, there were a few patients who were less than 40 years when their
AAE-C1-INH started. Among these were 3 patients with anti-C1-INH autoantibodies but no MGUS or malignant lymphoma. We conclude, therefore, that a diagnosis of AAE-C1-INH cannot be ruled out in patients less than 40 years old.

We found that AAE-C1-INH was associated with a variety of disorders including MGUS, malignant non-Hodgkin lymphoma, anti-C1INH autoantibodies, and other conditions. In some patients, there was no associated disorder identifiable. A total of $27.3 \%$ patients had an underlying lymphoma and in $75.0 \%$ of those patients the lymphoma was detected by monitoring the signs of AAE-C1-INH. This underscores the importance of early diagnosis of AAE-C1-INH. We also report that $25.0 \%$ of patients had a low-grade malignant lymphoma and approximately half of them had a splenic marginal cell lymphoma. This confirms that splenic marginal cell lymphoma is the most common type of underlying lymphoma in patients with AAE-C1-INH [23, 24].

Table 5 Dose-dependent treatment effects in acquired angioedema C1-inhibitor patients without $(N=6)$ and with $(N=8)$ antiC1-INH autoantibodies

\begin{tabular}{|c|c|c|c|c|c|c|c|c|c|c|c|c|}
\hline \multirow[b]{2}{*}{$\begin{array}{l}\text { pdC1- } \\
\text { INH } \\
\text { dose } \\
\text { (U) }\end{array}$} & \multicolumn{6}{|c|}{$\begin{array}{l}\text { Patients without autoantibodies against } \mathrm{C} 1-\mathrm{INH} \\
(\mathrm{N}=6)\end{array}$} & \multicolumn{6}{|c|}{$\begin{array}{l}\text { Patients with autoantibodies against C1-INH } \\
(N=8)\end{array}$} \\
\hline & $\begin{array}{l}\text { No. of } \\
\text { patients }\end{array}$ & $\begin{array}{l}\text { No. of } \\
\text { attacks }\end{array}$ & $\begin{array}{l}\text { Effectively } \\
\text { treated } \\
\text { patients }\end{array}$ & $\begin{array}{l}\text { Effectively } \\
\text { treated } \\
\text { attacks }\end{array}$ & $\begin{array}{l}\text { Non- } \\
\text { effectively } \\
\text { treated } \\
\text { patients }\end{array}$ & $\begin{array}{l}\text { Non- } \\
\text { effectively } \\
\text { treated } \\
\text { attacks }\end{array}$ & $\begin{array}{l}\text { No. of } \\
\text { patients }\end{array}$ & $\begin{array}{l}\text { No. of } \\
\text { attacks }\end{array}$ & $\begin{array}{l}\text { Effectively } \\
\text { treated } \\
\text { patients }\end{array}$ & $\begin{array}{l}\text { Effectively } \\
\text { treated } \\
\text { attacks }\end{array}$ & $\begin{array}{l}\text { Non- } \\
\text { effectively } \\
\text { treated } \\
\text { patients }\end{array}$ & $\begin{array}{l}\text { Non- } \\
\text { effectively } \\
\text { treated } \\
\text { attacks }\end{array}$ \\
\hline 500 & 3 & 338 & 3 & 337 & 1 & 1 & 7 & 102 & 5 & 33 & 3 & 69 \\
\hline 1000 & 4 & 8 & 3 & 7 & 1 & 1 & 6 & 907 & 5 & 900 & 3 & 7 \\
\hline 1500 & 0 & 0 & 0 & 0 & 0 & 0 & 2 & 4 & 1 & 1 & 1 & 3 \\
\hline 2000 & 0 & 0 & 0 & 0 & 0 & 0 & 4 & 315 & 3 & 311 & 1 & 4 \\
\hline \multirow[t]{2}{*}{3000} & 0 & 0 & 0 & 0 & 0 & 0 & 1 & 1 & 1 & 1 & 0 & 0 \\
\hline & -. & 346 & - & 344 & 2 & 2 & - & 1329 & - & 1246 & - & 83 \\
\hline
\end{tabular}

Note: The same patients may be represented as effectively treated and non-effectively treated 
MGUS and non-Hodgkin lymphoma are both B-cell lymphoproliferative disorders. MGUS may transform into plasmocytoma, Waldenström's macroglobulinemia and other lymphoproliferative disorders. How lymphoproliferative disorders result in C1-INH deficiency is not exactly clear. Binding of C1-INH to the dysprotein of MGUS or directly to lymphoma tissue could lead to a low level of C1-INH. The role that anti-C1-INH autoantibodies play in C1-INH deficiency is less clear. A total of $11.4 \%$ of patients in our series had anti-C1-INH autoantibodies without MGUS, lymphoma or other associated disorder, even after a long observation period of 15.8 years. Our results show that MGUS may underlie AAE-C1-INH with and without anti-C1-INH autoantibodies and, also, that malignant lymphoma may underlie AAE-C1-INH in the presence or absence of dysproteins or anti-C1-INH autoantibodies. Some patients with AAE-C1-INH have only neutralizing autoantibodies to $\mathrm{C} 1-\mathrm{INH}$ and no other associated disorder. In the past, it was assumed that this was a separate type of AAE-C1-INH (AAE-C1-INH type 2) [25, 26]. The younger age at onset of angioedema described here could be a special feature of this subgroup of patients. However, the small number of patients needs to be considered. At present, it is not clear whether lymphoproliferative disorders (MGUS and lymphoma) and anti-C1-INH autoantibodies have one common pathogenic mechanism leading to $\mathrm{C} 1-\mathrm{INH}$ deficiency.

Our results show that pdC1-INH reduces the average attack duration by more than $60 \%$. It is highly effective in resolving attacks, as evaluated by attack and by patient analysis, most notably if treatment is administered early in an attack. According to patients' self-assessment, all patients responded well to pdC1-INH in nearly all of their attacks.

It has been reported that a few AAE-C1-INH patients have needed treatment with high doses of pdC1-INH or that some patients have become completely or partially resistant to this treatment [4]. Some of these patients had autoantibodies to C1-INH concentrate [1, 27]. In contrast, our results show that the vast majority (93.8\%) of attacks in patients with anti-C1-INH autoantibodies responded well to pdC1-INH and that the response rate was similar to patients without anti-C1-INH autoantibodies (99.4\% of attacks). Therefore, we conclude that patients with anti-C1-INH antibodies can respond to pdC1-INH. However, in some patients with anti-C1-INH autoantibodies, doses to effectively treat attacks need to be more than double that in patients without autoantibodies. In our series, 4 out of 8 patients with anti-C1-INH autoantibodies did not respond sufficiently to pdC1-INH treatment in some attacks, whereas in other attacks in the same patients, treatment with pdC1-INH was effective. A variation in the level of
anti-C1-INH autoantibodies may be a reason for this. Repeated and long-term investigations of the anti-C1-INH autoantibody levels in AAE-C1-INH patients are needed to explain the relationship between autoantibody level and treatment dose.

Given the low prevalence of the disorder, this is a relatively large observational study examining the clinical characteristics of AAE-C1-INH. The study recruited a considerable number of patients and recorded the details of a large number of attacks treated with pdC1-INH over a long period of time. The study is limited by its observational and retrospective study design involving data retrieval from patient reports, which may have resulted in some bias. The fact that not all patients could be tested for anti-C1-INH autoantibodies also limits the results.

\section{Conclusions}

In summary, our study demonstrated that the clinical symptoms of AAE-C1-INH are similar to those of HAE-C1-INH. Early diagnosis of AAE-C1-INH is important because of the risk of asphyxiation by laryngeal edema and because AAE-C1-INH is frequently associated with an underlying malignant disorder. AAE-C1-INH attacks can be treated with pdC1-INH, which is fast-acting and is highly effective in nearly all AAE-C1-INH attacks, including those in patients with anti-C1-INH autoantibodies.

\section{Methods \\ Patients}

Patients for this observational study were followed up at the AOSM from March 1986 to August 2017. Diagnosis of AAE-C1-INH was based on personal history of recurrent angioedema, no family history of angioedema and plasma examination of C1-INH, C4 and C1q. The absence of a C1-INH (SERPING1) genetic mutation confirmed diagnosis in some patients. The study was approved by the local ethics committee (Ethics Committee of the Landesärztekammer Rheinland-Pfalz, 837.413.13 (9098-F)) and all patients gave their informed consent to participate in the study.

\section{Study design}

The clinical health records of 44 patients with AAE-C1-INH were reviewed retrospectively and patient characteristics noted. Thirty-two of these patients were treated for acute attacks with pdC1-INH. The efficacy variables were defined as follows: (1) the duration by which attacks were shortened after treatment, (2) the time between injection and resolution of symptoms, and (3) patient assessed treatment efficacy. The duration of treated attacks was compared with the duration of untreated attacks in the same individuals. Untreated attacks were defined as those which occurred before the first administration of pdC1-INH. Treated attacks were defined 
as those treated with pdC1-INH. Attack duration was defined as the time between attack onset and resolution of symptoms. Data on the time to injection and the type and duration of the attacks was recorded using standardized questionnaires. The course of the attacks was divided into first relief from symptoms and complete resolution of symptoms. In addition, patients evaluated the treatment effectiveness themselves during interviews and/or documented it in a patient diary. The following treatment evaluations were recorded by patients: treatment effective (responders) and treatment not or not sufficiently effective (non-responders).

\section{Treatment}

The treatment group received intravenous, pasteurized pdC1-INH concentrate (Berinert ${ }^{\circ}$ (CSL Behring, Marburg, Germany)) which was either self-injected or administered by the patient's general practitioner, at their local hospital or at our department. The usual dose comprised $500 \mathrm{U}$ of pdC1-INH, which corresponds to a C1-INH plasma activity of about $500 \mathrm{~mL}$ of fresh plasma. For treatment of skin swellings, $500 \mathrm{U}$ pdC1-INH was recommended. If the patient had a body weight over $80 \mathrm{~kg}$ or if the patient felt that the $500 \mathrm{U}$ dose was not sufficiently effective, $1000 \mathrm{U}$ pdC1-INH was administered. Patients were counseled that mild skin swellings should not be treated. Mild skin swellings were defined as swellings limited to the back of one hand or foot or swellings at one extremity or at the trunk with a diameter of $<20 \mathrm{~cm}$. Treatment was recommended for all facial and genital swellings. In skin swellings of the extremities, treatment was only recommended when the swellings were $>20 \mathrm{~cm}$ in diameter or if the whole extremity was affected or when the swelling of one extremity was followed by a swelling of another extremity or parts of the trunk within $24 \mathrm{~h}$. Doses higher than $1000 \mathrm{U}$ were administered only if the clinical response was not sufficient. All patients received vaccination for hepatitis $B$ virus.

\section{Laboratory methods}

C1-INH function was determined using the chromogenic substrate C2H5CO-Lys(e-Cbo)-Gly-Arg-pNA (Immunochrom C1-INH, Technoclone, Vienna, Austria). Normal functional levels of C1-INH were defined as $70-130 \%$. Antigenic levels of $\mathrm{C} 1-\mathrm{INH}, \mathrm{C} 4$ and $\mathrm{C} 1 \mathrm{q}$ were quantified by radial immunodiffusion. Plasma protein levels were considered normal if they fell within the following ranges: $\mathrm{C} 1-\mathrm{INH}=15.4-33.8 \mathrm{mg} / \mathrm{dL}, \quad \mathrm{C} 4=16.4-31.3 \mathrm{mg} / \mathrm{dL}$, and $\mathrm{C} 1 \mathrm{q}=0.1-0.25 \mathrm{~g} / \mathrm{L}$. Autoantibodies to $\mathrm{C} 1-\mathrm{INH}$ were measured as previously described [26].

\section{Statistical analysis}

\section{Patient characteristics}

The following parameters were analyzed with univariate statistics (mean and SD) or frequency tables, as appropriate, for all patients and patients treated with pdC1-INH: baseline and demographic characteristics, associated disorders, monoclonal gammopathy antibodies, anti-C1-INH autoantibodies, prior and concomitant treatment, number of stays at hospital due to angioedema, number of stays in the intensive care unit, number of intubations, number of untreated swellings, and the average duration of untreated attacks. Univariate statistics for C1-INH function, C1-INH protein, C4 and $\mathrm{C} 1 \mathrm{q}$ at first visit in AOSM were also generated for all patients and for patients treated with pdC1-INH.

\section{Plasma-derived C1 inhibitor concentrate treatment}

Univariate statistics for single doses of pdC1-INH were applied for both per-attack analysis and per-patient (on average doses) analysis. The number of attacks treated with pdC1-INH, the duration of pdC1-INH treatment, and the age at last pdC1-INH injection were analyzed with univariate statistics in a per-patient analysis. The number of attacks at different sites and the number of attacks treated with different doses of pdC1-INH were analyzed with a frequency table and frequency graph statistics in a per-attack analysis. In addition, a per-patient analysis was generated to show the number patients who preferably (i.e. in $90 \%$ or more of their attacks) received a special single dose of pdC1-INH.

For the time between onset of attack and injection, the time between injection and first sign of efficacy, the time between injection and complete resolution of symptoms, and the average shortening of attacks with treatment, univariate statistics were generated both in a per-patient and in a per-attack analysis.

\section{Efficacy variables}

The original dataset included data on the average duration of attacks with and without treatment with pdC1-INH for each patient as well as the number of attacks for each patient. The primary analysis on duration of attack was, therefore, a per-patient analysis which provided statistics on the average attack durations over all treated patients. In addition, a descriptive analysis on a per-attack basis was done by weighting the individual average duration of attacks by the individual number of attacks (untreated and treated).

Univariate statistics were applied for the average duration of attack treated with pdC1-INH and for the average duration of untreated attacks in both a per-patient and in a per-attack analysis. For the average shortening of the duration of attacks the following statistics were generated: univariate statistics, a two-sided Wilcoxon signed rank test (hypothesis: no shortening), and a 90\% $\mathrm{CI}$ in a per-patient analysis.

In order to investigate the association between the time between injection and resolution of symptoms and 
the time between onset of attack and injection, linear regression analysis with graphical representation was generated. A similar regression analysis was performed for the association between average shortening of attacks and the time to injection, the association between shortening of attacks and the preferred single dose and the association between time to resolution of symptoms and the preferred single dose.

The percentage of effectively treated attacks, as assessed by the patient, was calculated for the total number of treated attacks, anti-C1-INH antibody positive patients, anti-C1-INH antibody positive patients with MGUS, and anti-C1-INH antibody negative patients. All analyses were performed using Excel or SAS.

\section{Abbreviations}

AAE-C1-INH: acquired angioedema due to C1-inhibitor deficiency; AOSM: angioedema outpatient clinic at the Department of Dermatology, University of Mainz, Germany; C1-INH: C1 inhibitor; Cl: confidence interval; HAE: hereditary angioedema; MGUS: monoclonal gammopathy of undetermined significance; pdC1-INH: plasma-derived C1-INH concentrate

\section{Acknowledgments}

Editorial support was provided by Claire Kilmartin from Trilogy Writing and Consulting $\mathrm{GmbH}$. Statistical analysis was provided by Heinz-Otto Keinecke from Clinipace WW.

\section{Funding}

This work was supported by an unrestricted educational grant from CSL Behring.

\section{Availability of data and materials}

The datasets used and/or analyzed during the current study are available from the corresponding author on reasonable request.

\section{Authors' contributions}

KB: Defined the concept and design of the study, acquired, analysed and interpreted the data and wrote the manuscript. PS: Participated in the design of the study, acquired the data, collected samples, and critically revised the manuscript. JH: Defined the concept and design of the study, performed statistical analysis and drafted the manuscript. All authors have read and approved the final version of the manuscript.

\section{Ethics approval and consent to participate}

The study was approved by the Ethics Committee of the Landesärztekammer Rheinland-Pfalz, 837.413 .13 (9098-F). All patients gave their informed consent to participate in the study.

\section{Consent for publication}

Not applicable.

\section{Competing interests}

The authors declare that they have no competing interests.

\section{Publisher's Note}

Springer Nature remains neutral with regard to jurisdictional claims in published maps and institutional affiliations.

\section{Author details}

${ }^{1}$ Department of Dermatology, Johannes Gutenberg University, Langenbeckstr. 1, 55131 Mainz, Germany. ${ }^{2}$ Department of Medical Psychology and Medical Sociology, Johannes Gutenberg University, Mainz, Germany.
Received: 11 October 2018 Accepted: 5 March 2019

Published online: 13 March 2019

\section{References}

1. Zanichelli A, Azin GM, Wu MA, Suffritti C, Maggioni L, Caccia S, et al. Diagnosis, course, and management of angioedema in patients with acquired C1-inhibitor deficiency. J Allergy Clin Immunol. 2017;5:1307-13.

2. Bouillet-Claveyrolas L, Ponard D, Drouet C, Massot C. Clinical and biological distinctions between type I and type II acquired angioedema. Am J Med. 2003;115:420-1.

3. Dobson G, Edgar D, Trinder J. Angioedema of the tongue due to acquired C1 esterase inhibitor deficiency. Anaesth Intensive Care. 2003;31:99-102.

4. Cicardi M, Zanichelli A. Acquired angioedema. Allergy Asthma Clin Immunol. 2010;6:14

5. Castelli R, Zanichelli A, Cicardi M, Cugno M. Acquired C1-inhibitor deficiency and lymphoproliferative disorders: a tight relationship. Crit Rev Oncol Hematol. 2013;87:323-32.

6. Cicardi M, Beretta A, Colombo M, Gioffré D, Cugno M, Agostoni A. Relevance of lymphoproliferative disorders and of anti-C1 inhibitor autoantibodies in acquired angio-oedema. Clin Exp Immunol. 1996;106: 475-80

7. Donaldson VH, Wagner CJ, Davis AE 3rd. An autoantibody to C1-inhibitor recognizes the reactive center of the inhibitor. J Lab Clin Med. 1996;127: 229-32.

8. Mandle R, Baron C, Roux E, Sundel R, Gelfand J, Aulak K, et al. Acquired C1 inhibitor deficiency as a result of an autoantibody to the reactive center region of C1 inhibitor. J Immunol. 1994;152:4680-5.

9. Craig TJ, Bernstein JA, Farkas H, Bouillet L, Boccon-Gibod I. Diagnosis and treatment of bradykinin-mediated angioedema: outcomes from an angioedema expert consensus meeting. Int Arch Allergy Immunol. 2014;165: 119-27.

10. Cicardi M, Suffritti C, Perego F, Caccia S. Novelties in the diagnosis and treatment of angioedema. J Investig Allergol Clin Immunol. 2016;26:212-21.

11. Longhurst HJ, Zanichelli A, Caballero T, Bouillet L, Aberer W, Maurer M, et al. Comparing acquired angioedema with hereditary angioedema (types $\mathrm{I} / \mathrm{II}$ ): findings from the icatibant outcome survey. Clin Exp Immunol. 2017;188: 148-53.

12. Germenis $A E$, Speletas M. Genetics of hereditary angioedema revisited. Clin Rev Allergy Immunol. 2016;51:170-82.

13. Bork K, Wulff K, Hardt J, Witzke G, Staubach P. Hereditary angioedema caused by missense mutations in the factor XII gene: clinical features, trigger factors, and therapy. J Allergy Clin Immunol. 2009;124:129-34.

14. Bork K, Wulff K, Steinmuller-Magin L, Braenne I, Staubach-Renz P, Witzke G, et al. Hereditary angioedema with a mutation in the plasminogen gene. Allergy. 2018;73:442-50.

15. Bafunno V, Firinu D, D'Apolito M, Cordisco G, Loffredo S, Leccese A, et al. Mutation of the angiopoietin-1 gene (ANGPT1) associates with a new type of hereditary angioedema. J Allergy Clin Immunol. 2018;141:1009-17.

16. Bork K. An evidence based therapeutic approach to hereditary and acquired angioedema. Curr Opin Allergy Clin Immunol. 2014;14:354-62.

17. Lang DM, Aberer W, Bernstein JA, Chng HH, Grumach AS, Hide M, et al. International consensus on hereditary and acquired angioedema. Ann Allergy Asthma Immunol. 2012;109:395-402.

18. Bygum $A$, Vestergaard $H$. Acquired angioedema--occurrence, clinical features and associated disorders in a Danish nationwide patient cohort. Int Arch Allergy Immunol. 2013;162:149-55.

19. Jolles S, Williams P, Carne E, Mian H, Huissoon A, Wong G, et al. A UK national audit of hereditary and acquired angioedema. Clin Exp Immunol. 2014;175:59-67.

20. Bork K, Meng G, Staubach P, Hardt J. Hereditary angioedema: new findings concerning symptoms, affected organs, and course. Am J Med. 2006;119: 267-74.

21. Kőhalmi KV, Veszeli N, Cervenak L, Varga L, Farkas H. A novel prophylaxis with C1-inhibitor concentrate in hereditary angioedema during erythema marginatum. Immunol Lett. 2017;189:90-3.

22. Bygum A, Broesby-Olsen S. Rapid resolution of erythema marginatum after icatibant in acquired angioedema. Acta Derm Venereol. 2011;91:185-6.

23. Gobert D, Paule R, Ponard D, Levy P, Frémeaux-Bacchi V, Bouillet $L$, et al. A nationwide study of acquired $\mathrm{C} 1$-inhibitor deficiency in France: characteristics and treatment responses in 92 patients. Medicine (Baltimore). 2016;95:e4363. 
24. Castelli R, Wu MA, Arquati M, Zanichelli A, Suffritti C, Rossi D, et al. High prevalence of splenic marginal zone lymphoma among patients with acquired C1 inhibitor deficiency. Br J Haematol. 2016;172:902-8.

25. Jackson J, Sim RB, Whelan A, Feighery C. An IgG autoantibody which inactivates C1-inhibitor. Nature. 1986;323:722-4.

26. Alsenz J, Bork K, Loos M. Autoantibody-mediated acquired deficiency of C1 inhibitor. N Engl J Med. 1987;316:1360-6.

27. Alsenz J, Lambris JD, Bork K, Loos M. Acquired C1 inhibitor (C1-INH) deficiency type II. Replacement therapy with C1-INH and analysis of patients' C1-INH and anti-C1-INH autoantibodies. J Clin Invest. 1989;83: $1794-9$

Ready to submit your research? Choose BMC and benefit from:

- fast, convenient online submission

- thorough peer review by experienced researchers in your field

- rapid publication on acceptance

- support for research data, including large and complex data types

- gold Open Access which fosters wider collaboration and increased citations

- maximum visibility for your research: over $100 \mathrm{M}$ website views per year

At $\mathrm{BMC}$, research is always in progress.

Learn more biomedcentral.com/submissions 